\title{
The Impact of Stimulus Presentation and Size on Preference
}

\author{
James W. Moore ${ }^{1} \cdot$ Keith C. Radley $^{1} \cdot$ Evan H. Dart $^{1} \cdot$ Heather M. Whipple $^{1} \cdot$ \\ Emily J. Ness ${ }^{1} \cdot$ Ashley N. Murphy ${ }^{1} \cdot$ Chris Furlow $^{1} \cdot$ Joy K. Wimberly $^{1} \cdot$ Ashley Smith $^{1}$
}

Published online: 21 September 2016

(C) Association for Behavior Analysis International 2016

\begin{abstract}
The impact of stimulus size and presentation on choice during a preference assessment was investigated using a modified multiple-stimulus without replacement (MSWO) technique. Stimuli were either presented with a uniform magnitude, as determined by mass, or in a manner consistent with caregiver report of reinforcer consumption. While both assessment procedures identified the same top three preferred items in three out of five cases, greater variability in the preference rank of less preferred items was observed between assessments.
\end{abstract}

Keywords Preference assessment $\cdot$ Choice $\cdot$ Reinforce magnitude $\cdot$ Caregiver report

Since the introduction of stimulus preference assessments over 30 years ago, a large body of research has emerged to refine not only procedural issues with implementation but also to clarify the parameters that may influence responding during such assessments (e.g., Pace, Ivanic, Edwards, Iwata, \& Page, 1985; Wacker, Berg, Wiggins, Muldoon, \& Cavanaugh, 1985; see DeRosa \& Roane, 2015, for a detailed review). More recently, Steinhilber and Johnson (2007) compared the effects of brief and relatively long access to items included in a preference assessment. Their findings suggested that data collected during preference assessments may vary based on the amount of postselection duration of stimulus access. Studies such as this, and others (e.g., DeLeon, Frank, Gregory, \& Allman, 2009), continue to demonstrate the dynamic nature of reinforcing stimuli,

James W. Moore

james.moore@usm.edu

1 Applied Behavior Analysis Program, Department of Psychology, University of Southern Mississippi, 118 College Drive, Box 5025, Hattiesburg, MS 39406, USA as well as the influence of various motivating operations (MOs) on moment-to-moment preference and choice.

More recently, DeLeon et al. (2014) found that participants demonstrated a preference for accumulated reinforcers, rather than equally distributed amounts. From a practical standpoint, this raises a potential issue regarding the manner in which stimuli are presented during a preference assessment. For example, in an array of six edible items, the stimuli could be presented in an equated fashion (e.g., determined by total mass) or based on baseline reports of reinforcer consumption. These approaches may yield different types of stimulus control and MO influence that could alter both the individual preference for an item, and the overall hierarchy as well. No past research has offered data to guide practitioners in the presentation of edible stimuli within a preference assessment; therefore, it is important to understand how presentation format may impact choice behavior. The physical weight of items presented in the array could be one of many variables that could influence differential responding within a preference assessment. Parameters related to physical characteristics of the stimulus may yield stimulus control over responding that can be altered as the weight, volume, or other physical characteristic of the stimulus is manipulated. From a behavioral economics perspective, even the presence of other items in a stimulus array may influence responding (see Reed, Kaplan, \& Becirevic, 2015, for a review). In the current study, two variations of a six-item multiple-stimulus without replacement (MSWO) format were used to evaluate the impact of one physical characteristic, item weight, on participant choice.

\section{Method}

Five participants were selected from a summer social skills program at a local university. Katie was a 4-year-old female 
with no known diagnosis. Eliza was a 5-year-old female with a previous diagnosis of attention-deficit/hyperactivity disorder (ADHD). Grace was a 6-year-old female with no known diagnosis. Winston was a 6-year-old male with a previous diagnosis of autism spectrum disorder (ASD). Chris was a 9-yearold male with a previous diagnosis of ADHD. All participants used vocal speech, engaged in at least simple conversations, and could follow multistep instructions.

All sessions were conducted in a room $(6.5 \mathrm{~m}$ by $8.9 \mathrm{~m})$ within an academic building on a university campus. Six edible items were used in all preference assessments and were identified based on caregiver report (Fisher, Piazza, Bowman, \& Amari, 1996). Fisher et al.'s procedure was slightly modified to also determine the caregiver's report of a typical serving size of each edible. Caregivers were asked, "When your child eats (name of the item), how much does he/she usually eat at one time?" Furthermore, caregivers were asked how the edibles were typically presented. For example, some caregivers reported that they would give edible items in a receptacle of some sort, while others reported that they allowed their child to access the edible item in its natural packaging. Across all conditions, access to stimuli was restricted outside the experimental sessions and sessions were conducted after a period of at least 3 hours since the children had last eaten.

Experimental Design and Procedures In the current study, two preference assessments were conducted using a multielement design, with three repetitions of each preference assessment conducted for all participants. All procedures employed a multiple-stimulus without replacement (MSWO) method first reported by DeLeon and Iwata (1996). In the caregiver report MSWO, all items in the stimulus array were presented based on the average serving size amounts reported by the caregivers. One procedural variation from DeLeon and Iwata's procedure involved presentation of the stimulus array on a table rather than a tray. Each stimulus was placed 4 in. apart and $4 \mathrm{in}$. from the edge of the table facing the participant. Masking tape was used to mark the placement location of every item within the array. Winston, for example, was reported by his mother to prefer Laffy Taffy ${ }^{\mathrm{TM}}$ candy. Based on her report, it was determined that he typically would receive the entire bag of candy. So, during the caregiver report MSWO, an entire bag of Laffy Taffy ${ }^{\mathrm{TM}}$ was presented in the stimulus array. Additionally, stimuli were presented in the manner in which caregivers reported (e.g., in the original package, in a plastic bowl). While most caregivers reported presenting stimulus items in their original package, such as a whole bag of candy or an entire box of crackers, some reported presenting items in premeasured amounts in a bowl or storage bag. These parents did not use measurements of mass to determine amounts but rather reported filling the entire receptacle with the stimulus. In these examples, efforts were made to present the stimulus items exactly the way caregivers reported them delivered at home and other settings. Even though the items were presented in a way consistent with caregiver reports, access to the chosen items was still limited to $30 \mathrm{~s}$.

In the equal amount MSWO, each stimulus was presented as a 1 -g serving. Servings were weighed using a digital scale and prepared prior to sessions. While stimuli were equated based on raw mass, the 1-g weight was chosen to also produce a clear difference in apparent visual volume compared to the caregiver report stimuli. For instance, Winston received $1 \mathrm{~g}$ of a randomly selected Laffy Taffy ${ }^{\mathrm{TM}}$. This equated to roughly one-fourth of a single piece of the candy, as compared to an entire bag containing up to 10 pieces of candy. The two conditions were counterbalanced across subjects to minimize order effects. Winston, Eliza, and Grace experience the equal amount procedure first, while Chris and Katie encountered the caregiver report method first. The only procedural difference across both methods was the manner in which the stimuli were presented in the array.

Response Measurement, Reliability, and Procedural Integrity Item selection was defined and recorded whenever a participant touched any part of his hand to any one of the items in the stimulus array. Grams consumed was defined as the difference in weight of stimulus items prior to and then after an MSWO session. These data are available upon request. Interobserver agreement (IOA) was assessed in $100 \%$ of the sessions and was calculated on a trial-by-trial basis. Agreement for item selections was calculated by dividing the number of agreements (including both occurrence and nonoccurrence) by the total number of trials and multiplying by $100 \%$. IOA across all sessions was $97 \%$. Procedural integrity was assessed across $100 \%$ of the conditions and was calculated by dividing the total number of correct steps followed by the total number of steps possible and multiplying by $100 \%$. Procedural integrity averaged $99 \%$ across all sessions.

Data Analysis Results from each MSWO produced ranked preference hierarchies based on the percentage of times each item was selected when presented in the array across caregiver report and equal amount MSWO assessments. Hierarchies were calculated using the methods described by DeLeon and Iwata (1996). Consumption data were calculated by subtracting the post-session stimulus amount in grams by the pre-session amount. Additionally, Kendall's rank correlations were calculated between caregiver report and equal amount MSWO assessments for each participant.

\section{Results and Discussion}

As displayed in Fig. 1, divergent results were found not only in the percentage of item selection but also in the rank order of 
Fig. 1 Percentage of item selection in the caregiver report (dark bars) and the equal amount (light bars) methods for all five participants

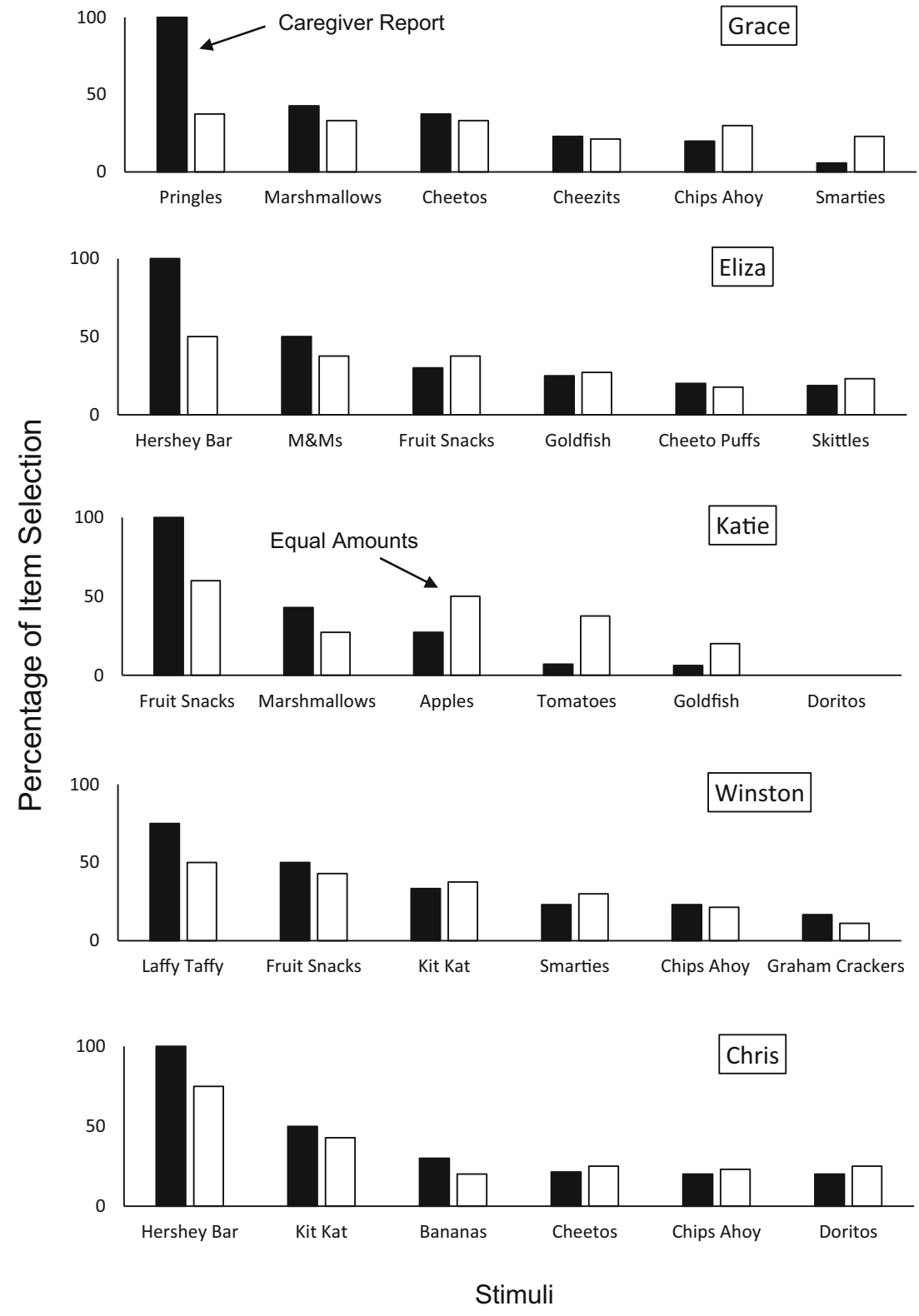

the hierarchies themselves (see Table 1 for a comparison). For all five participants, the caregiver report method led to the emergence of one preferred stimulus. Conversely, a higher degree of parity was found across stimuli when the equal amount method was employed. For example, Grace (top panel) chose Pringles $100 \%$ of the time during the caregiver report method, establishing it as the most preferred stimulus, with marshmallows falling second with an item selection percentage around $43 \%$. During the equal amount method, however, Pringles were selected only $37.5 \%$ of the time, with marshmallows and Cheetos falling less than $4 \%$ behind at $33 \%$. Across all participants, the equal amount method provided a larger array of potential reinforcers than results obtained using the caregiver report method. Table 1 summarizes rank order across both procedures. Table 2 displays the grams consumed across sessions for each MSWO preference assessment for each participant. Interestingly, in some cases, the equal amount procedure occasioned higher consumption than the caregiver report despite the fact that participants had access to a higher amount of the stimulus in the caregiver report condition. For example, Winston consumed more Laffy Taffy $^{\text {TM }}$ in the equal amount condition. As can be seen on Table 2, this difference was noted across some stimulus items for each participant. Table 3 presents the rank-order correlations between each MSWO preference assessment for each participant. Winston was the only participant whose preference assessment data yielded identical hierarchies across MSWO methods. Eliza and Katie's data produced significant rank correlations, suggesting that there was a high but imperfect level of correspondence between the two hierarchies. The 
Table 1 Rank-order standings for all stimuli in the caregiver report (CG rank) and equal amount (EA rank) across all participants

\begin{tabular}{|c|c|c|}
\hline Item & CG rank & EA rank \\
\hline \multicolumn{3}{|l|}{ Winston } \\
\hline Laffy Taffy & 1 & 1 \\
\hline Fruit snacks & 2 & 2 \\
\hline Kit Kat & 3 & 3 \\
\hline Smarties & 4 & 4 \\
\hline Chips Ahoy & 5 & 5 \\
\hline Graham crackers & 6 & 6 \\
\hline \multicolumn{3}{|l|}{ Eliza } \\
\hline Hershey bar & 1 & 1 \\
\hline $\mathrm{M} \& \mathrm{Ms}$ & 2 & 2 \\
\hline Fruit snacks & 3 & 3 \\
\hline Goldfish & 4 & 4 \\
\hline Cheeto Puffs & 5 & 6 \\
\hline Skittles & 6 & 5 \\
\hline \multicolumn{3}{|l|}{ Katie } \\
\hline Fruit snacks & 1 & 1 \\
\hline Marshmallows & 2 & 4 \\
\hline Apples & 3 & 2 \\
\hline Tomatoes & 4 & 3 \\
\hline Goldfish & 5 & 5 \\
\hline Doritos & 6 & 6 \\
\hline \multicolumn{3}{|l|}{ Chris } \\
\hline Hershey bar & 1 & 1 \\
\hline Kit Kat & 2 & 2 \\
\hline Bananas & 3 & 6 \\
\hline Cheetos & 4 & 3 \\
\hline Chips Ahoy & 5 & 5 \\
\hline Doritos & 6 & 4 \\
\hline \multicolumn{3}{|l|}{ Grace } \\
\hline Pringles & 1 & 1 \\
\hline Marshmallows & 2 & 3 \\
\hline Cheetos & 3 & 2 \\
\hline Cheezits & 4 & 6 \\
\hline Chips Ahoy & 5 & 4 \\
\hline Smarties & 6 & 5 \\
\hline
\end{tabular}

remaining two participants' (i.e., Chris and Grace) MSWO data yielded preference hierarchies that were more disparate across assessment methods.

Although both methods produced the same top stimulus for all participants, there were important differences that should be noted. The caregiver report method produced a much flatter hierarchy, perhaps suggesting to practitioners that multiple stimuli could be used as potential reinforcers. In the equal amount method, the top stimulus dominated the array in such a way that practitioners may discount the utility of other stimuli. Future research should explore how such variance among
Table 2 Total grams consumed for all stimuli in the caregiver report (CG consumed) and equal amount (EA consumed) across all participants

\begin{tabular}{|c|c|c|}
\hline Item & CG consumed & EA consumed \\
\hline \multicolumn{3}{|l|}{ Winston } \\
\hline Laffy Taffy & 0.20 & 0.50 \\
\hline Fruit snacks & 0.70 & 0.50 \\
\hline Kit Kat & 0.43 & 0.50 \\
\hline Smarties & 0.03 & 0.13 \\
\hline Chips Ahoy & 0.13 & 0.37 \\
\hline Graham crackers & 0.10 & 0.17 \\
\hline \multicolumn{3}{|l|}{ Eliza } \\
\hline Hershey bar & 0.27 & 0.27 \\
\hline $\mathrm{M} \& \mathrm{Ms}$ & 0.07 & 0.23 \\
\hline Fruit snacks & 0.07 & 0.23 \\
\hline Goldfish & 0.03 & 0.17 \\
\hline Cheeto Puffs & 0.03 & 0.10 \\
\hline Skittles & 0.07 & 0.00 \\
\hline \multicolumn{3}{|l|}{ Katie } \\
\hline Fruit snacks & 0.87 & 0.50 \\
\hline Marshmallows & 0.43 & 0.37 \\
\hline Apples & 0.57 & 0.50 \\
\hline Tomatoes & 0.43 & 0.50 \\
\hline Goldfish & 0.13 & 0.20 \\
\hline Doritos & 0.00 & 0.00 \\
\hline \multicolumn{3}{|l|}{ Chris } \\
\hline Hershey bar & 0.33 & 0.60 \\
\hline Kit Kat & 0.27 & 0.53 \\
\hline Bananas & 0.40 & 0.60 \\
\hline Cheetos & 0.07 & 0.10 \\
\hline Chips Ahoy & 0.23 & 0.37 \\
\hline Doritos & 0.10 & 0.13 \\
\hline \multicolumn{3}{|l|}{ Grace } \\
\hline Pringles & 0.20 & 0.55 \\
\hline Marshmallows & 0.10 & 0.60 \\
\hline Cheetos & 0.38 & 0.42 \\
\hline Cheezits & 0.08 & 0.51 \\
\hline Chips Ahoy & 0.42 & 0.60 \\
\hline Smarties & 0.11 & 0.34 \\
\hline
\end{tabular}

Table 3 Kendall's rank correlations between both MSWO hierarchies across participants

\begin{tabular}{lc}
\hline Participant & Correlation \\
\hline Winston & $1.00^{*}$ \\
Chris & .467 \\
Eliza & $.867^{*}$ \\
Grace & .600 \\
Katie & $.733^{*}$ \\
\hline
\end{tabular}

*Statistically significant correlations (i.e., $p<.05$ ) 
items could impact practitioner choices of reinforcer selection. Some practitioners may simply look at placement within the hierarchy, thus lessening the importance of overall preference, while others may value differences in preference across items in addition to hierarchical placement.

The current findings contribute both to the practice of administering preference assessments as well as basic information regarding stimulus preference. The current results may suggest that the purpose of the preference assessment should be considered when deciding on an appropriate presentation method. For example, if an intervention calls for a reinforcer of larger magnitude in which the practitioner must identify one or two powerful reinforcers, such as in a token economy, then the caregiver report may yield clinically relevant outcomes. If reinforcers are delivered in smaller, more frequent units, such as in intensive teaching interactions with initially rich schedules of reinforcement, then the equal amount method may be preferred. Also, these results preliminarily suggest that the equal amount method may identify a larger number of potential reinforcers. Future research should investigate the treatment utility of stimuli identified by each method.

The current study also suggests interesting conceptual points regarding the qualitative aspects of preferred stimuli. For example, data obtained across methods suggest that qualitative differences inherent in magnitude or continuity can influence value. This extends previous literature exploring factors that can impact reinforcer value (DeLeon et al., 2014; Steinhilber \& Johnson, 2007). Future research should explore comparisons of the "natural value" of a stimulus versus the "optimal value." In other words, does the difference in magnitude of a stimulus in the context of intervention sessions and how the individual consumes the stimulus in the natural environment matter? Also, how might consumption of the edible stimuli (putative reinforcers) be impacted by the tasks or demands in the intervention context. Readers are encouraged to refer Reed, Niileksela, and Kaplan (2013) for a review of behavioral economics related to practice.

The stimulus control exerted over presentation amount has both practical and conceptual value. It is possible that the value of a stimulus might be the greatest at a certain quantity, no more and no less, at which it is most customarily consumed (i.e., history and schedule of reinforcement). As such, when a stimulus is presented with a varying magnitude, this may also impact other unanticipated aspects of stimulus control exerted by the physical parameters of each stimulus. When considered as a physical stimulus unit, $1 \mathrm{oz}$ of marshmallows appears physically different than an entire bag of the same stimulus. Relational factors, such as "more than" and "less than" may also impact choice in ways that warrant further investigation. These results may suggest that other factors, such as relational networks, may, at least in part, contribute to choice during the procedures. Recently, research has suggested a three-element relational network in which a "small" stimulus may exude different stimulus control than "medium" and/or "large" stimuli (Whelan, Barnes-Holmes, \& Dymond, 2006). Given the preliminary nature of this study, future research should explore further variables that may interact related to choice behavior.

This study is limited by a number of factors. Most importantly, participant responding may have generalized from more typical settings during the caregiver report sessions due to the presentation format rather than size alone. For example, if a stimulus was presented in a manufacturer's package in the caregiver report sessions but in a neutral package for the equal amount sessions, then differential responding may have resulted from generalized stimulus control via the packaging. Future studies should present manipulated amounts in similar neutral packages to control for possible generalization effects.

Data obtained during each preference assessment were not verified with either a reinforcer assessment or used within an intervention protocol. As such, it is impossible to fully determine if the stimuli investigated would actually function as reinforcers. Additionally, by choosing to equate stimuli based on mass, other unintended factors may have contributed to item selection behavior. In an array in which all six stimuli weighed the same amount, some stimuli occupied more physical space than the others. For example, 1 oz of marshmallows occupies more physical area than $1 \mathrm{oz}$ of cookies. Due to this, participant choice may have been at least partly influenced by the visual presentation of the stimuli. Additional research should explore other presentation parameters to determine the potential stimulus control exuded by other factors, such as the physical area occupied by the stimulus. Additionally, past research on the general MSWO procedure includes quantitative comparison measures, such as the Kendall rank-order correlation coefficient (see DeLeon \& Iwata, 1996). Given the small number of participants and sessions, this study is limited by a lack of such comparisons. Future research may wish to run similar comparisons on a larger group of participants so that a Kendall coefficient can be determined.

Of note, none of the current participants presented major intellectual delays. Traditionally, other methods, such as interviews, are employed by practitioners in lieu of direct preference assessments. As such, it is currently unknown what types of results would be obtained if the current methodology was employed with individuals with major intellectual delays. Future research should explore outcomes of a caregiver report and equal amount comparison with individuals more typical of direct preference assessment methodologies.

Finally, the current study only utilized edible stimuli. The results of this study yield little information about how similar presentation methods with other stimuli, such as tangible stimuli, may lead to differential preference assessment results. In the future, researchers may wish to explore similar methods with other types of stimuli, including tangible and social 
stimuli. Further directions may include modifications of these procedures with tangible or social stimuli.

Compliance with Ethical Standards All aspects of the study complied with the ethical guidelines set forth by the Behavior Analyst Certification Board.

Informed Consent Informed consent was obtained from all individual participants included in the study.

Conflict of Interest The authors declare that they have no conflict(s) of interest.

\section{References}

DeLeon, I. G., \& Iwata, B. A. (1996). Evaluation of a multiple-stimulus presentation format for assessing reinforce preferences. Journal of Applied Behavior Analysis, 29, 519-533.

DeLeon, I. G., Chase, J. A., Frank-Crawford, M. A., Carreau-Webster, A. B., Bullock, C. E., \& Jennett, H. K. (2014). Distributed and accumulated reinforcement arrangements: evaluations of efficacy and preference. Journal of Applied Behavior Analysis, 47, 1-21.

DeLeon, I. G., Frank, M. A., Gregory, M. K., \& Allman, M. J. (2009). On the correspondence between preference assessment outcomes and progressive-ratio schedule assessments of stimulus value. Journal of Applied Behavior Analysis, 42, 729-733.
DeRosa, N. M., \& Roane, H. S. (2015). Preference and demand characteristics of reinforcement: practical extensions. In F. D. DiGennaro Reed \& D. D. Reed (Eds.), Autism service delivery: bridging the gap between science and practice. New York, NY: Springer.

Fisher, W. W., Piazza, C. C., Bowman, L. G., \& Amari, A. (1996). Integrating caregiver report with a systematic choice assessment to enhance reinforce identification. American Journal on Mental Retardation, 101, 15-25.

Pace, G. M., Ivancic, M. T., Edwards, G. L., Iwata, B. A., \& Page, T. J. (1985). Assessment of stimulus preference and reinforcer value with profoundly retarded individuals. Journal of Applied Behavior Analysis, 18, 249-255.

Reed, D. D., Kaplan, B. A., \& Becirevic, A. (2015). Basic research on the behavioral economics of reinforcer value. In F. D. DiGennaro Reed \& D. D. Reed (Eds.), Autism service delivery: bridging the gap between science and practice. New York, NY: Springer.

Reed, D. D., Niileksela, C. R., \& Kaplan, B. A. (2013). Behavioral economics: a tutorial for behavior analysis in practice. Behavior Analysis in Practice, 6, 34-54.

Steinhilber, J., \& Johnson, C. (2007). The effects of brief and extended stimulus availability on preference. Journal of Applied Behavior Analysis, 40, 767-772.

Wacker, D. P., Berg, W. K., Wiggins, B., Muldoon, M., \& Cavanaugh, J. (1985). Evaluation of reinforcer preferences for profoundly handicapped students. Journal of Applied Behavior Analysis, 18, 173-178.

Whelan, R., Barnes-Holmes, D., \& Dymond, S. (2006). The transformation of consequential functions in accordance with the relational frames of more-than and less-than. Journal of the Experimental Analysis of Behavior, 86, 314-335. 